\title{
Impurity Spin Magnetization of Thin Fe Doped Au Films
}

\author{
E. Seynaeve, K. Temst, F. G. Aliev,* and C. Van Haesendonck \\ Laboratorium voor Vaste-Stoffysica en Magnetisme, Katholieke Universiteit Leuven, Celestijnenlaan 200 D, B-3001 Leuven, Belgium \\ V. N. Gladilin, ${ }^{\dagger}$ V. M. Fomin $,^{\dagger}, \star$ and J. T. Devreese ${ }^{\ddagger} \S$ \\ Theoretische Fysica van de Vaste Stof, Departement Natuurkunde, Universiteit Antwerpen (UIA), Universiteitsplein 1, \\ B-2610 Antwerpen, Belgium \\ (Received 30 August 1999)
}

\begin{abstract}
In order to probe the influence of the surface-induced anisotropy on the impurity spin magnetization, we measure the anomalous Hall effect in thin AuFe films at magnetic fields up to $15 \mathrm{~T}$. The observed suppression of the anomalous Hall resistivity at low fields as well as the appearance of a minimum in the differential Hall resistivity at higher fields can be explained by our theoretical model, which takes into account the influence of a polycrystalline film structure on the surface-induced anisotropy. Our results imply that the apparent discrepancy between different experimental results for the size effects in dilute magnetic alloys can be linked to a different microstructure of the samples.
\end{abstract}

PACS numbers: 75.20.Hr, 75.30.Cr, 75.30.Gw, 73.50.Jt

In very dilute magnetic alloys, finite size effects may occur for sample dimensions comparable to the size of the Kondo screening cloud [1]. While some experiments [2] revealed a considerable decrease of the logarithmic Kondo anomaly in the resistivity of thin films and narrow wires already at the $\mu \mathrm{m}$ scale, other experiments [3] showed an almost constant Kondo anomaly for wire widths down to $40 \mathrm{~nm}$. Recent theoretical calculations indicated the complex, dynamical nature of the screening cloud, implying that the simple picture of a static, spherically symmetric screening cloud is not correct $[1,4]$.

Zawadowski et al. [5] linked the size dependent Kondo scattering to a surface-induced anisotropy of the magnetic impurity spins. Because of the interaction of the impurities with the conduction electrons, which suffer from the spin-orbit scattering by the nonmagnetic host atoms, the impurity spins tend to be aligned parallel to the sample boundaries [6]. The surface-induced anisotropy should remain active for more concentrated spin glass alloys. Finite size effects have indeed been observed in the resistivity of spin glasses with reduced dimensions $[7,8]$.

Finite size effects should also be observable in the impurity spin magnetization. Earlier magnetization experiments on single film [9] and on multilayered [10] spin glasses had linked a depression of the freezing temperature $T_{f}$ to a lower critical dimensionality for the spin glass transition. Here, we show that measuring the magnetic field dependence of the anomalous Hall effect in thin films of a AuFe spin glass [9] provides a powerful method to probe the influence of the surface-induced anisotropy on the $\mathrm{Fe}$ spin magnetization. At low magnetic fields, the magnetization signal related to the spin glass freezing is strongly suppressed when compared to the bulk behavior. The appearance of an extra magnetization signal at higher fields can be linked to a reorientation of impurity spins which are blocked by the surface-induced anisotropy at lower fields. The low field magnetization is further suppressed when moving the Fe doping towards the film surface. We have developed a theoretical model which takes into account the polycrystalline film structure. The presence of additional internal surfaces at the grain boundaries introduces a particular spatial dependence of the spin anisotropy which is essential for understanding the Hall effect data. Our model provides an explanation for the apparent discrepancies between the different experiments which have probed the size dependence of the Kondo and spin glass resistivity.

Thin films of AuFe alloys have been prepared by codeposition on oxidized silicon wafers of Au (99.9999\% purity) and $\mathrm{Fe}(99.99 \%$ purity) in an ultrahigh vacuum molecular beam epitaxy growth chamber. A multiterminal sample geometry is obtained by depositing the films through a contact mask. Apart from $30 \mathrm{~nm}$ thick Fe doped $\mathrm{Au}$ films and pure Au reference films, we have also prepared $\mathrm{Au} / \mathrm{AuFe} / \mathrm{Au}$ and $\mathrm{AuFe} / \mathrm{Au} / \mathrm{AuFe}$ trilayers. For the $30 \mathrm{~nm}$ thick trilayer samples, the central layer has a thickness of $15 \mathrm{~nm}$, while the two outer layers have a thickness of $7.5 \mathrm{~nm}$. The Hall resistance as well as the longitudinal resistance are measured with an ac resistance bridge in perpendicular magnetic fields up to $15 \mathrm{~T}$.

Adding Fe impurity spins to pure Au causes the appearance of a nonlinear anomalous Hall component. Figure 1(a) shows typical Hall effect data at $T=4.2 \mathrm{~K}$ which have been obtained by McAlister and Hurd [11] for polycrystalline pure bulk $\mathrm{Au}$ as well as for a 0.98 at. \% AuFe bulk spin glass. The anomalous Hall resistivity varies proportional to the $\mathrm{Fe}$ spin magnetization, and its temperature dependence at low fields reveals a sharp peak near the freezing temperature $T_{f}$. In agreement with direct magnetization measurements, the peak is washed out at higher magnetic fields [11,12].

As illustrated in Fig. 1(b), the anomalous Hall effect is much weaker in a thin 2 at. \% AuFe spin glass film when compared to the bulk material. Although the Fe concentration is about twice as large as in Fig. 1(a), deviations 

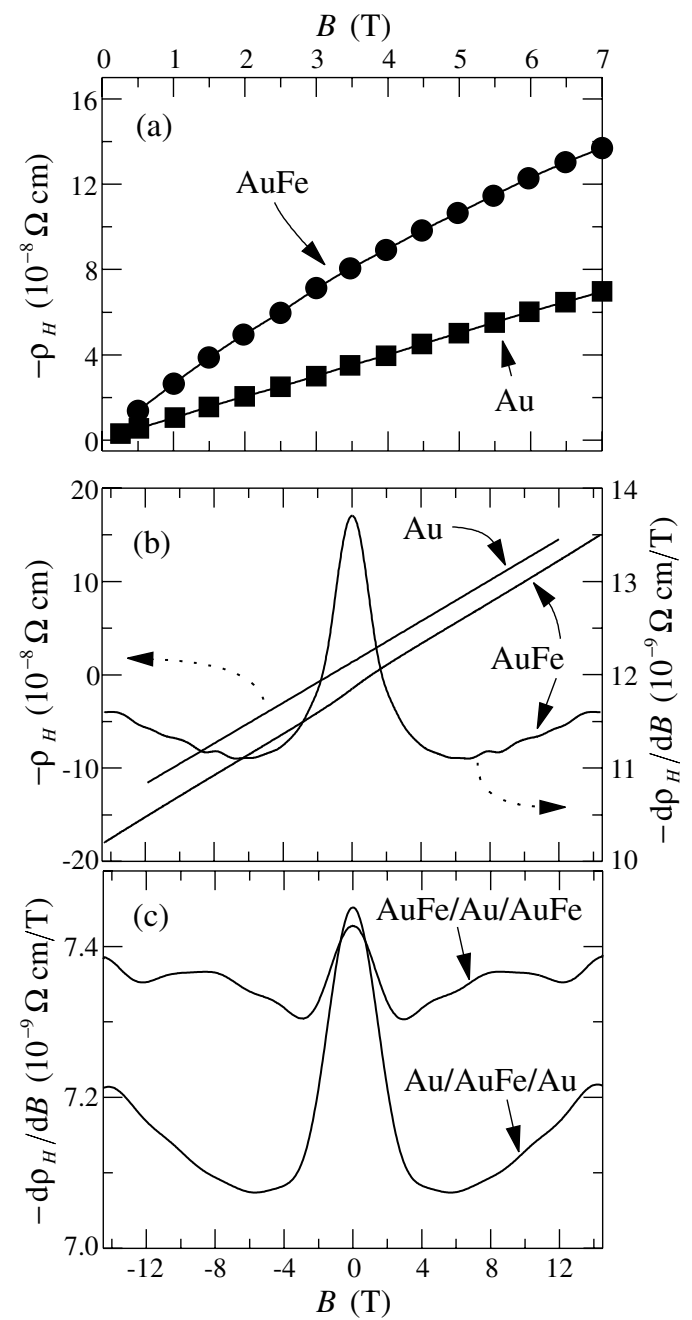

FIG. 1. (a) Magnetic field dependence of the Hall resistivity for a bulk polycrystalline Au sample and a bulk 0.98 at. \% AuFe sample at $T=4.2 \mathrm{~K}$ (from Ref. [11]). (b) Magnetic field dependence of the Hall resistivity for a pure Au film and for a 2 at. $\%$ AuFe film at $T=1 \mathrm{~K}$. Both films have a thickness of $30 \mathrm{~nm}$. For the AuFe film, the differential Hall resistivity $-d \rho_{H} / d B$ is also shown. (c) Magnetic field dependence of the differential Hall resistivity for the $\mathrm{Au} / \mathrm{AuFe} / \mathrm{Au}$ and $\mathrm{AuFe} / \mathrm{Au} / \mathrm{AuFe}$ trilayers (Fe concentration is 3.5 at. \% for the $\mathrm{AuFe})$ at $T=1 \mathrm{~K}$.

from the classical linear Hall resistivity of the pure $\mathrm{Au}$ film are very small. In order to make the nonlinear anomalous Hall component more clearly visible, we also plot in Fig. 1(b) the differential Hall resistivity $-d \rho_{H} / d B$. The initial variation of the differential Hall resistivity at low fields reflects the destruction of the spin glass state due to the alignment of the impurity spins. The magnetic response resulting from this alignment has been reduced by an order of magnitude when compared to the bulk material. As discussed in detail below, we can attribute the reduced amplitude of $-d \rho_{H} / d B$ at low fields as well as the appearance of a minimum at higher fields to the surface-induced anisotropy.

The strength of the anisotropy of an impurity spin is predicted to increase when the impurity approaches the sample surface [5]. Consequently, the anisotropy effects should be less pronounced for a $\mathrm{Au} / \mathrm{AuFe} / \mathrm{Au}$ trilayer when compared to a $\mathrm{AuFe} / \mathrm{Au} / \mathrm{AuFe}$ trilayer. This is confirmed in Fig. 1(c) for AuFe layers with an Fe concentration of 3.5 at. \%. Both samples have the same total thickness and the same Fe content. The samples also have a comparable resistivity, and the maximum in the low field $(\sim 0.01 \mathrm{~T})$ anomalous Hall resistivity [9] occurs at a comparable temperature $\left(T_{f} \simeq 5 \mathrm{~K}\right)$. Nevertheless, the nonlinear behavior is clearly enhanced for fields below $4 \mathrm{~T}$ when moving the $\mathrm{Fe}$ impurities away from the surface. The reduced amplitude of $-d \rho_{H} / d B$ for the trilayers when compared to a single AuFe film [see Fig. 1(b)] results from a shortcircuiting by the pure Au layer with a much lower resistivity.

In order to calculate the nonlinear impurity magnetization in polycrystalline AuFe films, we generalize the theory of the surface-induced anisotropy $[5,6]$ to the case of thin films consisting of small grains. In contrast to the method used in Ref. [5], our approach, introduced in Ref. [6] for wires of dilute magnetic alloys, allows one to treat the surface-induced anisotropy in mesoscopic samples with a more complicated shape. Here, we have derived the Hamiltonian $\mathcal{H}_{\text {an }}$, which describes the surface-induced anisotropy for an impurity spin in an isolated brick-shaped grain:

$$
\mathcal{H}_{\mathrm{an}}=\mathcal{A} \sum_{\alpha, \beta} \mathcal{B}_{\alpha \beta} S_{\alpha} S_{\beta} \quad(\alpha, \beta=x, y, z) .
$$

The $S_{\alpha}$ are the operators for the components of the impurity spin $\mathbf{S}$. In contrast to a semi-infinite sample [5], the strength of the anisotropy has to be described in terms of a matrix with elements $\mathcal{A} \mathcal{B}_{\alpha \beta}$. Moreover, the calculated matrix elements $\mathcal{B}_{\alpha \beta}$ are complicated functions of the dimensions $\left(a_{x}, a_{y}, a_{z}\right)$ of the grain and of the impurity position. The material dependent constant $\mathcal{A}$ should range between 0.01 and $1 \mathrm{eV}$ for dilute AuFe alloys [5,6]. Taking the $z$ axis parallel to the magnetic field, the magnetization of an $\mathrm{Fe}$ spin is given by

$$
\left\langle S_{z}\right\rangle=-\frac{1}{2 \mu_{\mathrm{B}} Z} \sum_{k=1}^{5} \exp \left(-\frac{\mathcal{E}_{k}}{k_{\mathrm{B}} T}\right) \frac{d \mathcal{E}_{k}}{d B},
$$

where $\mu_{\mathrm{B}}$ is the Bohr magneton, and $Z=$ $\sum_{k=1}^{5} \exp \left(-\mathcal{E}_{k} / k_{\mathrm{B}} T\right)$. The index $k=1, \ldots, 5$ labels the roots $\mathcal{E}_{k}$ of the secular equation,

$$
\left|\mathcal{H}_{S_{z}^{\prime} S_{z}}-\mathcal{E} \delta_{S_{z}^{\prime} S_{z}}\right|=0 \quad\left(S_{z}, S_{z}^{\prime}=-2,-1,0,1,2\right),
$$

with the Hamiltonian $\mathcal{H}=-2 \mu_{\mathrm{B}} S_{z} B+\mathcal{H}_{\text {an }}$.

In Fig. 2 the calculated differential magnetization $\left[d\left\langle S_{z}\right\rangle / d B\right]_{\mathrm{gr}}$ (the square brackets with subscript "gr" correspond to an average over impurity positions within a grain) is shown as a function of the magnetic field for different lateral dimensions of the grains. The height $a_{z}$ of the grains is fixed at $30 \mathrm{~nm}$, i.e., the thickness of the AuFe film shown in Fig. 1(b). In the limiting case 


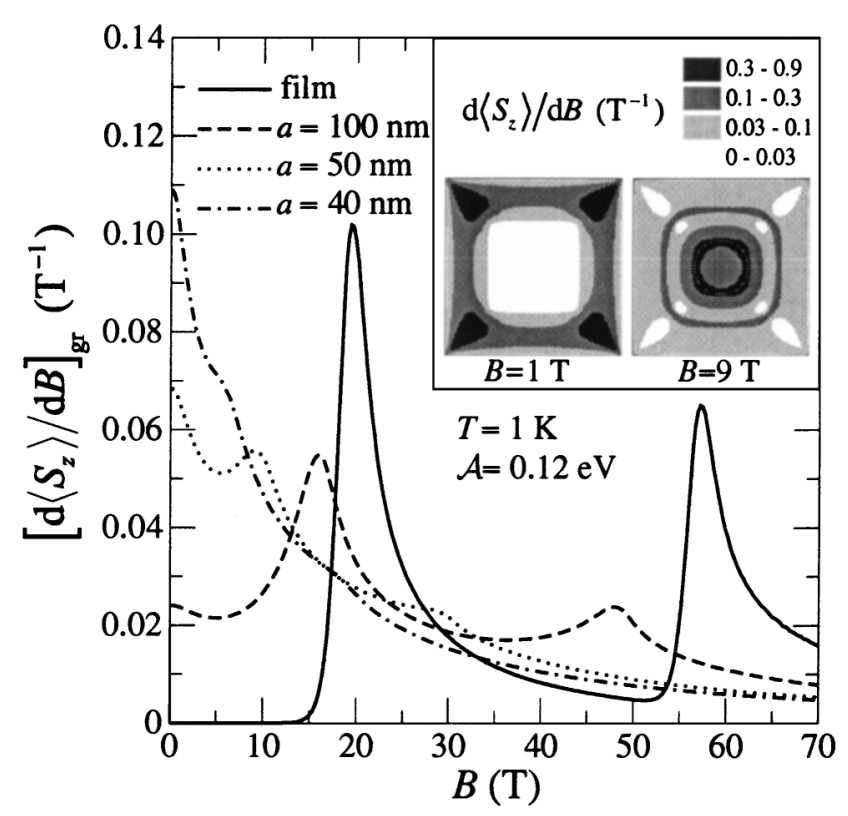

FIG. 2. Calculated magnetic field dependence of the differential magnetization $\left[d\left\langle S_{z}\right\rangle / d B\right]_{\mathrm{gr}}$ for grains with height $a_{z}=$ $30 \mathrm{~nm}$ and lateral dimensions $a_{x}=a_{y}=a$. Inset: Spatial distribution of the differential magnetization for a cross section perpendicular to the magnetic field at the center of a grain with $a=50 \mathrm{~nm}$.

of a single-crystal film $\left(a_{x}, a_{y} \rightarrow \infty\right)$, the impurity spin states at $B=0$ are known [5] to be the eigenstates of $S_{z}$, the energy eigenvalues being proportional to $S_{z}^{2}$. At low temperatures, only the state with $S_{z}=0$ will be populated. Hence, the impurity spin does not respond to a weak magnetic field. With increasing $B$ the energy level with $S_{z}=1$ becomes lower than that with $S_{z}=0$. This gives rise to the first peak in the field dependence of $\left[d\left\langle S_{z}\right\rangle / d B\right]_{\mathrm{gr}}$. The second peak appears when the state with $S_{z}=2$ becomes the ground state. The theoretical result for the single-crystal film in Fig. 2 clearly fails to describe our experimental results, since the calculated impurity spin magnetization remains zero at low magnetic fields (all spins are blocked parallel to the surface).

In Ref. [6 ], it was already shown that in narrow wires the presence of differently oriented surfaces leads to a rather intricate behavior of the impurity-spin anisotropy when compared to the case of a film. In brick-shaped grains, the competing influence of mutually perpendicular surfaces on the magnetic anisotropy gives rise to a partial cancellation of the anisotropy effect, resulting in a shift of the peaks in $\left[d\left\langle S_{z}\right\rangle / d B\right]_{\text {gr }}$ towards lower fields. Moreover, there exist specific locations within the grains where the magnetic anisotropy energy becomes negligibly small. At these locations the bulk behavior of the impurity spins is restored. Hence, a limited number of spins contribute to the spin glass freezing and are sensitive to very weak magnetic fields. In the inset of Fig. 2, we illustrate the large difference in the predicted response of spins located near the corners of the grains when compared to spins located more towards the center of the grains. For grains with lateral sizes larger than (but still comparable to) the height, our theoretical model predicts that a minimum in $\left[d\left\langle S_{z}\right\rangle / d B\right]_{\mathrm{gr}}$ can appear at relatively small magnetic fields. For those grains the initial part of the theoretical curves is similar to the measured differential Hall resistivity shown in Figs. 1(b) and 1(c). The aforementioned nonmonotonous behavior gradually disappears both in our experiments and in our theoretical model at temperatures well above the measured freezing temperature $T_{f}$. A consistent description of our anomalous Hall data requires one to assume a large value $\mathcal{A}=0.12 \mathrm{eV}$ in Eq. (1). This implies that a larger fraction of the alignment of the Fe spins by the magnetic field occurs at extremely high fields which are not accessible in the experiment.

Figure 3(a) shows the calculated field dependence of an average $\left[d\left\langle S_{z}\right\rangle / d B\right]_{\mathrm{cs}}$ over the positions of the impurities located within a cross section of a grain perpendicular to the magnetic field. The different curves correspond

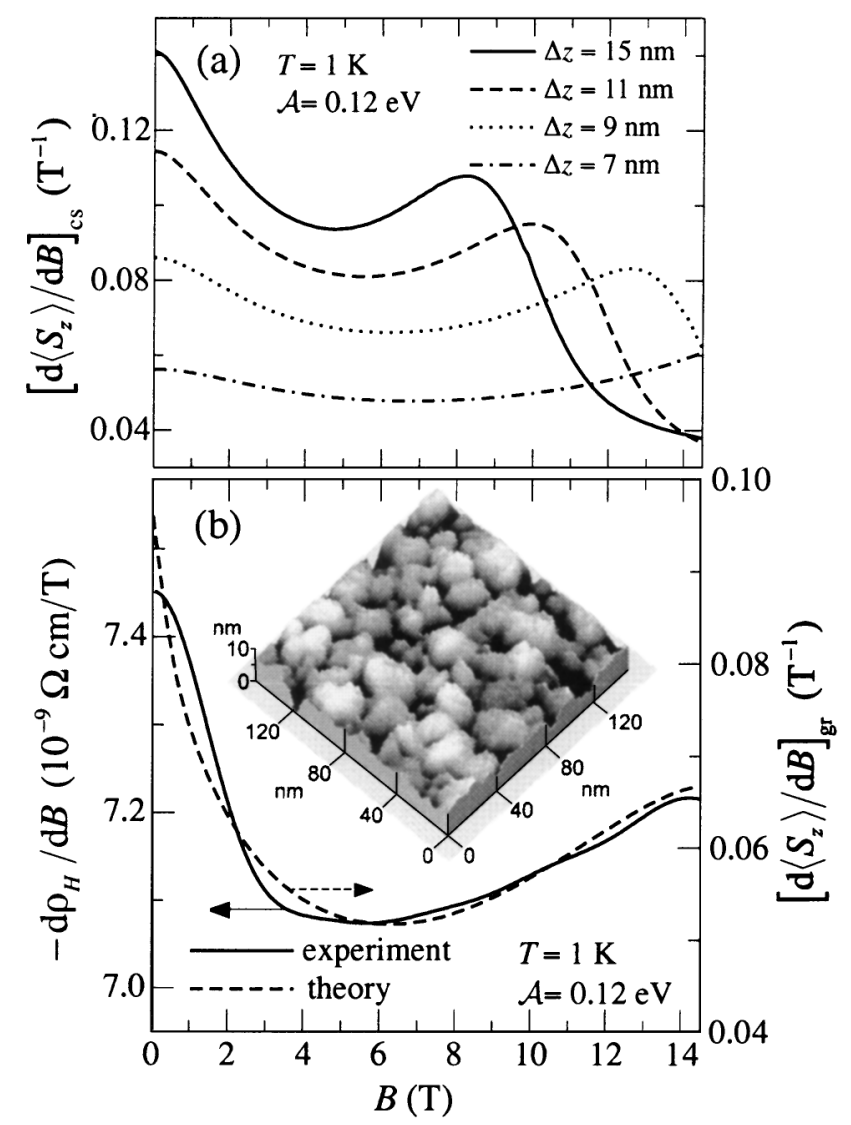

FIG.3. (a) Calculated magnetic field dependence of the differential magnetization $\left[d\left\langle S_{z}\right\rangle / d B\right]_{\mathrm{cs}}$ for a grain after averaging over a cross section perpendicular to the magnetic field. Different curves are for different distances $\Delta z$ to the top or bottom of the grain with a height of $30 \mathrm{~nm}$ and lateral size of $50 \mathrm{~nm}$. (b) Comparison between the differential Hall resistivity $-d \rho_{H} / d B$ measured for the $\mathrm{Au} / \mathrm{AuFe} / \mathrm{Au}$ trilayer (solid curve) and the calculated differential magnetization $\left[d\left\langle S_{z}\right\rangle / d B\right]_{\mathrm{gr}}$ for an ensemble of $\mathrm{Au} / \mathrm{AuFe} / \mathrm{Au}$ grains with a height of $30 \mathrm{~nm}$ and a thickness of the central AuFe layer of $15 \mathrm{~nm}$ (dashed curve). For the lateral grain size $a$, a lognormal distribution is assumed (see text). The inset shows an STM image of the surface of the $\mathrm{Au} / \mathrm{AuFe} / \mathrm{Au}$ trilayer. 
to different distances $\Delta z$ from the cross section to the top (or bottom) of the grain. The theoretical curves can be compared to the experimental field dependence of $-d \rho_{H} / d B$ for the $\mathrm{Au} / \mathrm{AuFe} / \mathrm{Au}$ and the $\mathrm{AuFe} / \mathrm{Au} / \mathrm{AuFe}$ trilayer shown in Fig. 1(c). The low field variation is much weaker for the AuFe/Au/AuFe trilayer. This is in agreement with our theoretical result that the surfaceinduced anisotropy becomes stronger for $\mathrm{Fe}$ spins which are closer to the top (or bottom) of a grain.

The inset of Fig. 3(b) shows a scanning tunneling microscopy (STM) image of the surface of the $\mathrm{Au} / \mathrm{AuFe} / \mathrm{Au}$ sample, revealing a rather broad distribution of grain sizes. In order to quantitatively fit the experimental data, we therefore calculate the magnetization for an ensemble of grains of different lateral size $a$, distributed lognormally with a statistical median $\bar{a}$ and a standard deviation $\sigma$. In Fig. 3(b), the measured differential Hall resistivity $-d \rho_{H} / d B$ for the $\mathrm{Au} / \mathrm{AuFe} / \mathrm{Au}$ trilayer is compared to the field dependence of $\left[d\left\langle S_{z}\right\rangle / d B\right]_{\mathrm{gr}}$, calculated for an ensemble of $\mathrm{Au} / \mathrm{AuFe} / \mathrm{Au}$ grains with $\bar{a}=80 \mathrm{~nm}$ and $\sigma=1.5$. We note that the value of $80 \mathrm{~nm}$ is larger than the typical grain size inferred from the STM images $(20 \mathrm{~nm})$. Our theoretical approach with isolated grains is strictly valid only when elastic defect scattering at the grain boundaries fully destroys the anisotropy which is caused by the conduction-electron-mediated interaction of an $\mathrm{Fe}$ spin with host atoms from neighboring grains. Using a larger grain size for the calculations allows one to take into account that a fraction of the electrons still moves ballistically between adjacent grains.

We conclude that our theoretical model for the surfaceinduced anisotropy of magnetic impurities in small metallic grains allows a quantitative description of the anomalous Hall resistivity in polycrystalline AuFe spin glass films. The small impurity spin magnetization at low magnetic field also provides an explanation for the small amplitude of the spin glass resistivity in thin AuFe films [8]. It is clear that the surface-induced anisotropy strongly affects the spin glass freezing, implying that the analysis of previous measurements, which investigated the reduction of the freezing temperature $T_{f}$ in thin films [9] and multilayers [10], should be revised. At this point it is not yet clear to what extent the observed effects are caused by an intrinsic size effect or if they can be totally accounted for by the surface-induced anisotropy.

For Kondo alloys with a small impurity content $(\sim 100 \mathrm{ppm})$, we are no longer able to measure the anomalous Hall effect. The surface-induced anisotropy should still cause a reduction of the impurity spin magnetization, resulting in a suppression of the Kondo resistivity. Earlier measurements of the Kondo resistivity in AuFe films confirmed the presence of such a suppression, which became weaker when covering the films with a pure Au layer [13]. Since the surface-induced blocking of a magnetic impurity spin is sensitive to the specific polycrystalline sample structure, the apparent discrepancy between different experimental results $[2,3,13]$ for the size dependence of the Kondo resistivity can be linked to a different microstructure of the samples.

The collaboration between the universities of Antwerpen and Leuven has been supported by the Fund for Scientific Research-Flanders (Belgium) as well as by the Belgian Interuniversity Attraction Poles research program (IUAP No. 4/10). Additional support has been obtained in Antwerpen and Leuven from the Flemish Concerted Action (GOA) research program and in Antwerpen from the Scientific Fund (BOF) of the Universiteit Antwerpen.

* Present address: Departamento de Física de la Materia Condensada, C-3, Universidad Autónoma de Madrid, E-28049 Madrid, Spain.

${ }^{\dagger}$ Permanent address: Department of Theoretical Physics, State University of Moldova, strada A. Mateevici 60, MD-2009 Kishinev, Republic of Moldova.

${ }^{\star}$ Also at Technische Universiteit Eindhoven, P.O. Box 513, NL-5600 MB Eindhoven, The Netherlands.

${ }^{\S}$ Also at Universiteit Antwerpen (RUCA), Groenenborgerlaan 171, B-2020 Antwerpen, Belgium.

[1] V. Barzykin and I. Affleck, Phys. Rev. Lett. 76, 4959 (1996); E. S. Sorensen and I. Affleck, Phys. Rev. B 53, 9153 (1996); V. Barzykin and I. Affleck, Phys. Rev. B 57, 432 (1998).

[2] G. Chen and N. Giordano, Phys. Rev. Lett. 66, 209 (1991); M. A. Blachly and N. Giordano, Phys. Rev. B 46, 2951 (1992); J. F. DiTusa, K. Lin, M. Park, M. S. Isaacson, and J. M. Parpia, Phys. Rev. Lett. 68, 678 (1992).

[3] V. Chandrasekhar, P. Santhanam, N. A. Penebre, R. A. Webb, H. Vloeberghs, C. Van Haesendonck, and Y. Bruynseraede, Phys. Rev. Lett. 72, 2053 (1994).

[4] G. Bergmann, Phys. Rev. Lett. 67, 2545 (1991); G. Bergmann, W. Shieh, and M. Huberman, Phys. Rev. B 46, 8607 (1992).

[5] O. Újsághy, A. Zawadowski, and B.L. Gyorffy, Phys. Rev. Lett. 76, 2378 (1996); O. Újsághy and A. Zawadowski, Phys. Rev. B 57, 11598 (1998); O. Újsághy and A. Zawadowski, Phys. Rev. B 57, 11609 (1998).

[6] V. M. Fomin, V. N. Gladilin, J. T. Devreese, C. Van Haesendonck, and G. Neuttiens, Solid State Commun. 106, 293 (1998).

[7] K. R. Lane, M. Park, M. S. Isaacson, and J. M. Parpia, Phys. Rev. B 51, 945 (1995).

[8] G. Neuttiens, J. Eom, C. Strunk, V. Chandrasekhar, C. Van Haesendonck, and Y. Bruynseraede, Europhys. Lett. 34, 617 (1996).

[9] H. Vloeberghs, J. Vranken, C. Van Haesendonck, and Y. Bruynseraede, Europhys. Lett. 12, 557 (1990).

[10] G. G. Kenning, J. M. Slaughter, and J.A. Cowen, Phys. Rev. Lett. 59, 2596 (1987); A. Gavrin, J. R. Childress, C. L. Chien, B. Martinez, and M. B. Salamon, Phys. Rev. Lett. 64, 2438 (1990).

[11] S. P. McAlister and C. M. Hurd, Solid State Commun. 19, 881 (1976).

[12] R. D. Barnard and I. Ul-Haq, J. Phys. F, Met. Phys. 18, 1253 (1988).

[13] M. A. Blachly and N. Giordano, Phys. Rev. B 51, 12537 (1995). 